\title{
A STOCHASTIC SH WAVE MODEL OF EARTHQUAKE GROUND MOTION
}

Takanori HARADA*

\begin{abstract}
The temporal and spatial variability of earthquake ground motions has been inferred experimentally using data from closely spaced seismograph arrays. In this paper, the earthquake ground motion varying in time-space domain is described analytically by the stochastic wave filtered by a surface soil layer of irregular thickness resting on half space. The SH wave is transmitted to the soil layer from the half space. The analytic expressions of the earthquake responses of irregular ground are based on the perturbation method, and their accuracy is examined by comparison with the responses computed by the direct boundary element method for many rough surface models consisting of topography with sinusoidal shape. Finally, an analytic expression for the frequency wavenumber spectrum of the stochastic wave at ground surface is then derived.
\end{abstract}

Key Words: ground motiom, earthquake, stochastic wave

\section{INTRODUCTION}

The spatial variation of earthquake ground motions is an important factor that should be carefully considered in the seismic design of buried lifelines such as tunnels and pipelines. The consideration of the spatial variation of ground motion may also have significant effects on the seismic response of structures with spatially extended foundations or multiple supports. In fact, for buried lifelines, the seismic deformation method was developed (Public Works Research Institute, 1977) and is now in practical use in Japan. For the seismic design of the Akashi Kaikyo Bridge foundations, a modified response spectrum was used taking into account the spatial variation of ground motions around the foundations (Kashima et al., 1984; Kawaguchi et al., 1987).

The temporal and spatial variability of earthquake ground motions has been inferred experimentally using data from closely spaced seismograph arrays. The SMART-1 array, for example, located at Lotung in the NE corner of Taiwan has provided valuable data for the analysis of ground motions in time-space domain. Numerous studies using the SMART-1 array data have been reported (Loh et al., 1982; Harada, 1984; Harada and Shinozuka, 1986; Harichandran, 1988; Abrahamson, 1985 and 1991). It is common in these studies that the accelerograms from each seismic event are described as samples from spacetime stochastic processes or stochastic waves and eventually the spatial coherence functions or the frequency wavenumber spectra are estimated.

\footnotetext{
* Department of Civil Engineering, Miyazaki University (Miyazaki, 889-21, Japan)
}

In this paper, the earthquake ground motion varying in time-space domain is described by the stochastic wave filtered by a single surface soil layer of irregular thickness, due to the irregular shape of the free-surface, resting on half space. The SH wave is transmitted to the soil layer from the half space. The analytic expressions of the earthquake responses of irregular ground are presented on the basis of the perturbation method, and their accuracy is examined by comparison with the responses computed by the direct boundary element method for many rough surface models consisting of topography with sinusoidal shape. Finally, an analytic expression for the frequency wavenumber spectrum of the stochastic wave at irregular ground surface is then derived.

The frequency wavenumber spectrum can be directly used for the digital simulation of the stochastic wave form, on the basis of the spectral representation method (Shinozuka et al., 1987). However, other definitions of correlation functions exist. In order to make clear those definitions as well as the relationships between other correlation functions and the frequency wavenumber spectrum, the following section describes a brief summary of the fundamentals of space-time correlation structure (Shinozuka et al., 1987).

\section{SPACE-TIME CORRELATION STRUCTURE}

In the stationary-homogeneous approach, it is well known that a stochastic wave $u_{0}(x, t)$ with zero mean value is characterized by the autocorrelation function defined as :

$$
R_{u_{0} u_{0}}(\xi, \tau)=E\left[u_{0}(x+\xi, t+\tau) u_{0}(x, t)\right]
$$




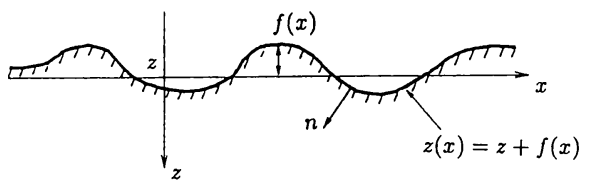

Fig.1 An irregular interface between two homogeneous media

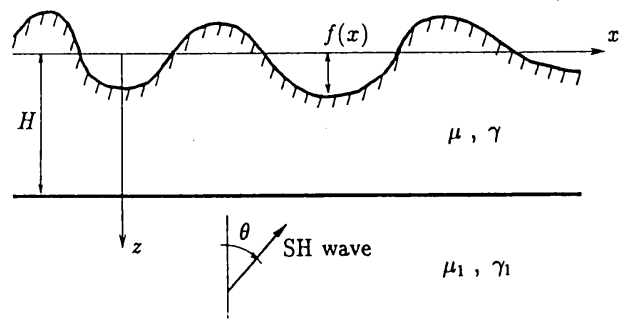

Fig.2 A single soil layer with irregular free-surface resting on half space

in which, $E[\cdot]$ denotes the expectation operator, $x$ is a vector of spatial coordinates $\left(x_{1}, x_{2}\right)$ and $t$ the time variable. Since the stochastic wave is assumed to be stationary in time and homogeneous in space, the autocorrelation function possesses the symmetric nature with respect to the origin :

$$
R_{u_{0} u_{0}}(\xi, \tau)=R_{u 0 u 0}(-\xi,-\tau)
$$

Assuming that the Fourier transform of the autocorrelation function exists, the frequency wavenumber spectrum of $u_{0}(x, t)$ is defined as :

$$
\begin{aligned}
S_{u_{0} u_{0}}(\kappa, \omega)= & \frac{1}{(2 \pi)^{3}} \iiint \\
& R_{u_{0} u_{0}} e^{-i(\kappa . \xi-\omega \tau)} d \xi_{1} d \xi_{2} d \tau
\end{aligned}
$$

and its inverse transform is given by :

$$
\begin{aligned}
R_{u_{0} u_{0}}(\xi, \tau)= & \iiint S_{u_{0} u_{0}}(\kappa, \omega) e^{i(x . \xi-\omega \tau)} \\
& d \kappa_{1} d \kappa_{2} d \omega \ldots \ldots \ldots \ldots \ldots \ldots \ldots \ldots \ldots \ldots \ldots
\end{aligned}
$$

The above two equations represent the Wiener Khintchine relations in three dimensions, where $\kappa$ is a vector of the wavenumbers $\left(\kappa_{1}, \kappa_{2}\right)$ and $\omega$ the frequency.

It can be shown from Eqs. (2) and (3a) that the frequency wavenumber spectrum is symmetric :

$$
S_{u_{0} u_{0}}(\kappa, \omega)=S_{u_{0} u_{0}}(-\kappa,-\omega)
$$

It is also shown according to Bochner (1956) that the frequency wavenumber spectrum is real and nonnegative :

$$
S_{u_{0} u_{0}}(\kappa, \omega) \geq 0
$$

It should be noted here that the autocorrelation function or the frequency wavenumber spectrum provide all information concerning the correlational characteristics of the stochastic wave $u_{0}(x, t)$. In fact other correlation functions can be defined using either the autocorrelation function or the frequency wavenumber spectrum. For example, the frequency-dependent autocorrelation function $C_{u_{0} u_{0}}(\xi, \omega)$ is defined as :

$$
\begin{aligned}
C_{u_{0} u_{0}}(\xi, \omega) & =\frac{1}{2 \pi} \int R_{u_{0} u_{0}}(\xi, \tau) e^{i \omega \tau} d \tau \\
& =\iint S_{u_{0} u_{0}}(\kappa, \omega) e^{i \alpha . \xi} d \kappa_{1} d k_{2} \cdots
\end{aligned}
$$

The frequency-dependent autocorrelation function is usually used to represent the correlational characteristics of stochastic wave because the correlation is in general decreased with separation distance and frequency. The frequency-dependent autocorrelation function represents directly such a correlation.

\section{SH WAVE FILTERED BY A SINGLE LAYER}

Response of a single layer with irregular surface resting on half space subjected to an $\mathrm{SH}$ wave as shown in Fig. 2 is considered in this section. The method is based on the perturbation approach (Kennett, 1972). In subsection 3.(1), the perturbation method by Kennett is summarized and then the response of the ground with irregular surface in Fig.2 is derived in subsection 3.(2).

\section{(1) Basic Equations for Ground with Irregular Interface}

For harmonic excitation with frequency $\omega$, the displacement wave field $v(x, z) \exp (-i \omega t)$ corresponding to $\mathrm{SH}$ wave propagations in a twodimensional homogeneous isotropic medium is known to be expressed as the matrix wave equation in the form (Kennett, 1972) :

$$
\frac{\partial}{\partial z} B(x, z)=A_{0}(z) B(x, z)
$$

where $B$ is the displacement-stress vector and $A_{0}$ is the operator matrix defined as :

$$
\begin{aligned}
& B_{S H}=\operatorname{col}\left[v, \tau_{y z}\right] \ldots \ldots \ldots \ldots \ldots \ldots \\
& A_{0 S H}=\left[\begin{array}{cc}
0 & \frac{1}{\mu} \\
-\mu \partial_{x x}-\rho \omega^{2} & 0
\end{array}\right]
\end{aligned}
$$

with

$$
\partial_{x}=\partial / \partial x, \partial_{x x}=\partial^{2} / \partial x^{2}
$$

where $\mu$ is the complex shear modulus, and $\rho$ the density of the medium.

Welded boundary conditions at the irregular interface (see Fig.1) require continuity of displacement and traction at each point on the interface. Therefore, a new displacement-stress vector $b$, being measured with respect to the local tangent plane at each point on the interface, has to be introduced. The new displacement-stress vector takes the form (Kennett, 1972) : 


$$
b(x, f)=\left(I+\frac{\partial f}{\partial x} Q_{0}\right) B(x, f)
$$

where $b$ and $B$ are evaluated along the irregular interface located at the depth $z(x)$ defined by :

$$
z(x)=z+f(x)
$$

with $z$ being the average depth of the interface and $f(x)$ being the lateral fluctuation of the interface (see Fig.1). In Eq.(10), $I$ is the unit matrix and $Q_{0}$ is given by.

$$
Q_{0 S H}=\left[\begin{array}{cc}
0 & 0 \\
-\mu \partial_{x} & 0
\end{array}\right]
$$

The irregular interface boundary condition can be expressed in the form :

$$
b_{1}(x, f)=b_{2}(x, f) \text {. }
$$

where subscripts indicate the respective media.

In order to obtain the approximation of Eq.(10), the scattered wave field $B(x, f)$ (displacementstress vector in the medium with irregular interface) is approximately related to the background field $B(x, z)$ (displacement-stress vector in the medium with horizontal plane interface) by using a Taylor's expansion around the average interface depth $z(x)=z$, and then substituting into Eq. (10), and omitting terms of order higher than $f$ and $\partial f / \partial x$, one obtains :

$$
b(x, f)=\left\{I+f A_{0}+\frac{\partial f}{\partial x} Q_{0}\right\} B(x, z)
$$

Furthermore, introducing the new notations, $b^{0}$ and $B^{0}$, which represent the background wave fields, and denoting the first-order approximations of $b$ and $B$ by $b^{\mathrm{I}}$ and $B^{\mathrm{I}}$, respectively, one obtains the first-order approximation of Eq.(14) as :

$$
b^{I}(x, f)=B^{I}(x, z)+\left\{f A_{0}+\frac{\partial f}{\partial x} Q_{0}\right\} B^{0}(x, z)
$$

with the following condition because $b$ equals to $B$ at the interface $z(x)=z$ in the case of horizontal plane interface $(f=0)$ :

$$
b^{0}(x, z)=B^{0}(x, z)
$$

The Fourier transform of Eq.(15) with respect to $x$, using the result that the Fourier transform of a product is the convolution of the Fourier transform, yields :

$$
\begin{aligned}
& b^{I}(\kappa, f)=B^{I}(\kappa, z) \\
& \quad+\int f\left(\kappa-\kappa^{\prime}\right) J\left(\kappa, \kappa^{\prime}\right) B^{0}\left(\kappa^{\prime}, z\right) d \kappa^{\prime} \cdots
\end{aligned}
$$

where

$$
J\left(\kappa, \kappa^{\prime}\right)=A_{0}\left(\kappa^{\prime}\right)+i\left(\kappa-\kappa^{\prime}\right) Q_{0}\left(\kappa^{\prime}\right)
$$

with

$$
J_{S H}=\left[\begin{array}{cc}
0 & \frac{1}{\rho C_{s}^{2}} \\
\rho C_{s}^{2} \kappa \kappa^{\prime}-\rho \omega^{2} & 0
\end{array}\right]
$$

In Eq.(19), $C_{s}$ is the complex $\mathrm{S}$-wave velocity given by :

$$
C_{s}=C_{s}^{0}\left(1-i D_{s}\right)
$$

with $C_{s}^{0}$ being the elastic $\mathrm{S}$-wave velocity and $D_{s}$ being the ratio of the linear hysteretic damping for S-wave.

The irregular interface boundary condition given by Eq.(13) can be written for the first-order approximations in frequency wavenumber domain as :

$$
B_{1}^{I}(\kappa, z)=B_{2}^{I}(\kappa, z)+S(\kappa, z)
$$

where

$$
\begin{aligned}
& S(\kappa, z)=\int_{-\infty}^{\infty} f\left(\kappa-\kappa^{\prime}\right) L_{21}\left(\kappa, \kappa^{\prime}\right) B_{2}^{0}\left(\kappa^{\prime}, z\right) d \kappa^{\prime} \\
& \text {............................... (21b) } \\
& L_{21}=J_{2}-J_{1}=-L_{12}
\end{aligned}
$$

Equation (21a) indicates that the presence of irregular interface results in discontinuity in the scattered wave field $B(x, z)$ at $z(x)=z$. This discontinuity acts like a seismic source $S$ which can be evaluated directly from the background wave field.

\section{(2) Response of Ground with Irregular Free Surface}

Response of a single soil layer with irregular free surface resting on half space subjected to incident SH wave as shown in Fig.2 is considered. For a free surface the traction has to vanish, so that for an irregular free surface $z(x)=f(x),(z=0)$, the scattered $\mathrm{SH}$ wave field takes the form :

$$
b(\kappa, f)=B(\kappa, f)=\operatorname{col}[U(\kappa, f), 0]
$$

Then, the first-order approximation of the interface boundary condition given by Eq.(17) can be expressed as :

$$
\begin{aligned}
& B^{I}(\kappa, f)=B^{I}(\kappa, 0) \\
& \quad+\int_{-\infty}^{\infty} f\left(\kappa-\kappa^{\prime}\right) J\left(\kappa, \kappa^{\prime}\right) B^{0}\left(\kappa^{\prime}, 0\right) d \kappa^{\prime} \cdots .
\end{aligned}
$$

Making use of the propagator matrix $P\left(\kappa, z, z_{0}\right)$ which satisfies (Kennett, 1972)

$$
\begin{aligned}
& \frac{\partial}{\partial z} P\left(\kappa, z, z_{0}\right)=A_{0}(\kappa, z) P\left(\kappa, z, z_{0}\right) \\
& P^{-1}\left(\kappa, z, z_{0}\right)=P\left(\kappa, z_{0}, z\right)
\end{aligned}
$$

the displacement-stress vector at the bedrock, $z(x)$ $=H$, can be transferred to that at the free surface, $z(x)=0$, such as :

$$
B(\kappa, 0)=P(\kappa, 0, H) B(\kappa, H)
$$

For an $\mathrm{SH}$ wave the propagator matrix is given by :

$$
\begin{aligned}
& P_{S H}\left(\kappa, z, z_{0}\right) \\
& \quad=\left[\begin{array}{cc}
\cos \gamma\left(z-z_{0}\right) & \frac{\sin \gamma\left(z-z_{0}\right)}{\mu \gamma} \\
-\mu \gamma \sin \gamma\left(z-z_{0}\right) & \cos \gamma\left(z-z_{0}\right)
\end{array}\right] .
\end{aligned}
$$


where $\gamma$ is the veritical ( $z$ axis) wavenumber given by :

$$
\gamma=\sqrt{\left(\frac{\omega}{C_{s}}\right)^{2}-\kappa^{2}} ; I_{m}(\gamma) \geq 0
$$

In order for the physical condition of a wave propagating in the positive direction of the vertical axis $(z$ axis) to be zero at infinity, then the imaginary part of the vertical wavenumber $I_{m}(\gamma)$ in Eq. (26b) must be positive.

It is assumed now that an input earthquake motion is an inclined SH wave with the amplitude $v_{\text {in }}$ and the horizontal wavenumber $\kappa_{0}$. Then, the input motion (represented by displacement-stress vector ) at the bedrock is expressed in the form :

$$
B\left(\kappa^{\prime}, H\right)=B\left(\kappa_{0}, H\right) \delta\left(\kappa^{\prime}-\kappa_{0}\right)
$$

where $\delta$ is the Delta function, and $\kappa_{0}$ is given by :

$$
\kappa_{0}=\frac{\omega \sin \theta}{C_{s}}
$$

in which $\theta$ is the angle of the incident $\mathrm{SH}$ wave, measured clockwise from the vertical axis as shown in Fig.2. For vertical incidence, $\theta=0^{\circ}$, then $\kappa_{0}=0$ from Eq.(27b). The input motion $B$ at the bedrock is related to the incident $\mathrm{SH}$ wave such that :

$$
B(\kappa, H)=R(\kappa) v_{0}(\kappa, H)
$$

where $v_{0}$ is the displacement vector in half space and $R$ is a transform matrix from the displacement vector to the displacement-stress vector which are defined as :

$$
\begin{aligned}
& v_{0}=\operatorname{col}\left[v_{\text {in }}, v_{\text {out }}\right] \ldots \ldots \\
& R=\left[\begin{array}{cc}
1 & 1 \\
i \mu_{1} \gamma_{1} & -i \mu_{1} \gamma_{1}
\end{array}\right]
\end{aligned}
$$

in which $v_{\text {out }}$ is the displacement amplitude of the outgoing $\mathrm{SH}$ wave in half space.

Substituting Eqs.(25) and (27) into (23), one obtains :

$$
\begin{aligned}
& B^{I}(\kappa, f)=P(\kappa, 0, H) B^{I}(\kappa, H) \delta\left(\kappa-\kappa_{0}\right) \\
& +f\left(\kappa-\kappa_{0}\right) J\left(\kappa, \kappa_{0}\right) P\left(\kappa_{0}, 0, H\right) B^{0}\left(\kappa_{0}, H\right)
\end{aligned}
$$

By considering the boundary conditions in which a) traction vanishes at the free surface and $b$ ) the incident SH wave is specified at the bedrock, these, together with the relationship of Eq.(28a), then Eq.(29) can be more explicitly expressed in the partitioned form :

$$
\begin{aligned}
& {\left[\begin{array}{c}
U^{I}(\kappa, f) \\
0
\end{array}\right]=\left[\begin{array}{ll}
F_{11} & F_{12} \\
F_{21} & F_{22}
\end{array}\right]\left[\begin{array}{c}
v_{\text {in }}(\kappa, H) \\
v_{\text {out }}^{I}(\kappa, H)
\end{array}\right] \delta\left(\kappa-\kappa_{0}\right)} \\
& +f\left(\kappa-\kappa_{0}\right)\left[\begin{array}{ll}
J_{11} & J_{12} \\
J_{21} & J_{22}
\end{array}\right]\left[\begin{array}{cc}
F_{11}^{0} & F_{12}^{0} \\
F_{21}^{0} & F_{22}^{0}
\end{array}\right]\left[\begin{array}{c}
v_{\text {in }}\left(\kappa_{0}, H\right) \\
v_{\text {out }}^{0}\left(\kappa_{0}, H\right)
\end{array}\right]
\end{aligned}
$$

where $F_{i j}$ represents the $(i j)$ th component of the matrix $P R$ and $F_{i j}^{0}=F_{i j}\left(\kappa_{0}, 0, H\right)$. In Eq.(30), the relation $v_{\text {in }}^{I}(\kappa, H)=v_{\text {in }}(\kappa, H)$ is used because the incident motion displacement is specified at the bedrock. From Eq.(30), one can obtain the scattered wave field $U^{I}(\kappa, f)=v^{I}(\kappa, f)$ at irregular free surface such as :

$$
\begin{aligned}
v^{I}(\kappa, f)= & {\left[\frac{p}{\cos \gamma H} \delta\left(\kappa-\kappa_{0}\right)\right.} \\
& +f\left(\kappa-\kappa_{0}\right) q \gamma_{0} \frac{\sin \gamma H}{\cos \gamma_{0} H \cdot \cos \gamma H} \\
& \left.\left(\frac{\kappa \kappa_{0}-\kappa_{0}^{2}}{\gamma \gamma_{0}}-\frac{\gamma_{0}}{\gamma}\right)\right] 2 v_{\text {in }} \ldots \ldots \ldots \ldots
\end{aligned}
$$

where

$$
\begin{aligned}
& p=\frac{1}{1+i \frac{\mu \gamma}{\mu_{1} \gamma_{1}} \frac{\sin \gamma H}{\cos \gamma H}} \cdots \\
& q=p_{0} \frac{1-i \frac{\mu \gamma}{\mu_{1} \gamma_{1}} \frac{\cos \gamma H}{\sin \gamma H}}{1+i \frac{\mu \gamma}{\mu_{1} \gamma_{1}} \frac{\sin \gamma H}{\cos \gamma H}}
\end{aligned}
$$

In Eq.(31a), the first term of the right-hand side represents the response displacement at free surface with a horizontal plane surface, and the second term the scattered wave displacement due to irregular free surface.

\section{ACCURACY OF THE PERTURBA- TION METHOD}

Since the analytic expression in Eq.(31) is based on the perturbation method, it is necessary to examine the accuracy and to reveal the practical limits imposed on Eq.(31). For this purpose, the response displacements at free surface are compared with those derived by the direct boundary element method. Four ground models are used in this comparison, each having the free surface topography of sinusoidal shapes with different heights and wave lengths. The accuracy of the direct boundary element method used in this comparison is described in subsection 4.(1), and then, the accuracy of the perturbation method is examined in subsection 4.(2).

\section{(1) Accuracy of the DBEM}

The boundary element method used in this study is based on the formulation (Wong and Jennings, 1975).

The frequency responses of the two-dimensional semicylindrical canyon and hill as shown in Fig.3 are computed by the boundary element method, and are compared with the exact solutions (Trifunac, 1973; Men and Yuan, 1992). Fig.3 shows the distribution of boundary elements along the surface of a canyon and hill used in this study. A total of seventy-one constant elements are used 
$\cdot 3 \mathrm{a}$

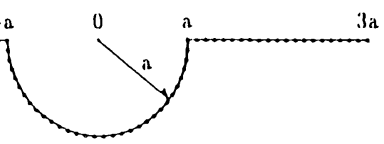

Fig.3 (a) Distribution of boundary elements along the surface of a semicylindrical canyon (71 constant elements are used)

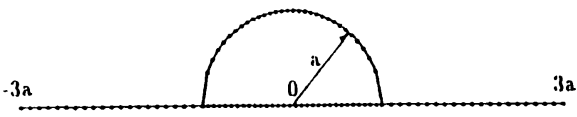

Fig.3 (b) Distribution of boundary elements along the surface of a semicylindrical hill (71 constant elements are used)

for calculation. Fig.4 shows an example on the frequency response distribution along the surface in and around the canyon by using discrete points (boundary element method), together with the exact solutions (Trifunac, 1973) using solid curves. Only the case for the dimensionless frequency $\omega a / \pi C_{s}=1.0$ is shown, where one half of the Swave length is equal to the radius $a$ of the canyon. Note that this is the dimensionless frequency $\eta$ in Trifunac's paper. The horizontal axis is the dimensionless space coordinate $x / a$, and the vertical axis represents the amplitude of frequency response relative to the incident wave amplitude $v_{i n}$. In Fig. 4 the comparisons are shown for four different incidence angles $\theta\left(0^{\circ}, 30^{\circ}, 60^{\circ}\right.$ and $\left.90^{\circ}\right)$. For the same conditions in the comparisons using a semicylindrical canyon in Fig.4, the comparisons using a semicylindrical hill are also performed. The results of comparison between the boundary element method and the exact solution (Men and Yuan, 1992) are shown in Fig.5. Excellent agreements are observed from Figs. 4 and 5 in the results by both methods, indicating the accuracy of the boundary element method used in this study.

(2) Accuracy of the Perturbation Method

The analytic expression in Eq.(31) for the response of the irregular free surface to the incident SH wave may be applied to the response of a semicylindrical canyon and hill used in subsection 4 (1) for examining the accuracy of the boundary element method. However the direct comparisons between the perturbation result in Eq.(31) and the exact solutions for a semicylindrical canyon and hill are not possible. The numerical Fourier transform is necessary for comparisons because the perturbation result in Eq.(31) is expressed in the frequencywavenumber domain $(\omega-\kappa)$, while the results of the boundary element method and the exact solutions for a canyon and hill are expressed in the frequency-space domain $(\omega-x)$. In order to perform the direct comparison between the perturbation method and the boundary element

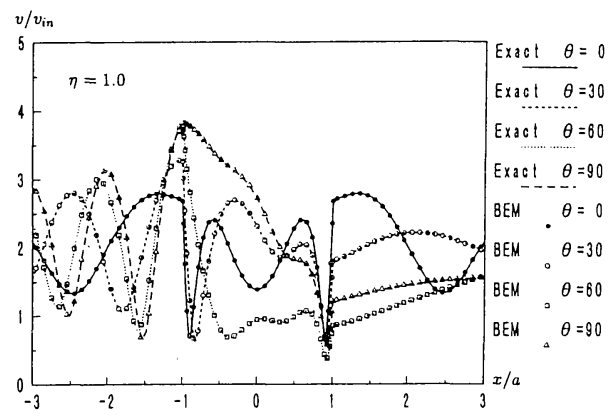

Fig.4 Comparisons in the frequency responses of semicylindrical canyon computed by boundary element method (discrete points) and the exact solution (lines) by Trifunac (1973)

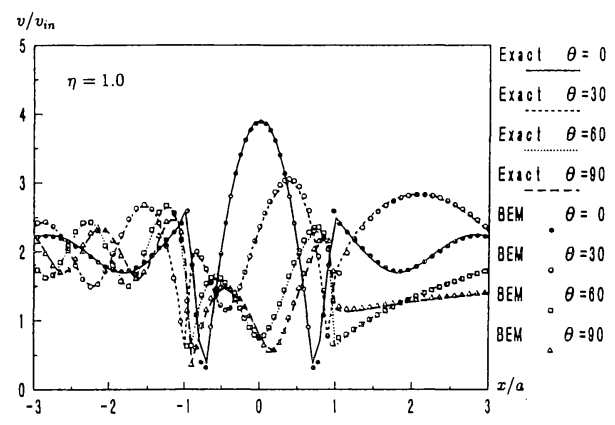

Fig.5 Comparisons in the frequency responses of semicylindrical hill computed by boundary element method (discrete points) and the exact solution (lines) by Yuan and Men (1992)

method without using the numerical Fourier transform, the frequency responses to incident $\mathrm{SH}$ wave are compared for the half space ground models with irregular free surface consisting of sinusoidal shapes having four different heights and wave lengths. The four half space ground models are shown in Fig.6, and the free surface heights and wave lengths in each model are indicated in Table 1.

For the half space ground model with irregular free surface consisting of sinusoidal shapes, the analytic expression of Fourier transform is possible, and then the response displacement in frequency-space domain with $\kappa_{0}=0$ can be obtained as :

$$
\begin{aligned}
& v^{I}(x, f(x), \omega)=\int_{-\infty}^{\infty} v^{I}(\kappa, f(\kappa)) e^{i \kappa x} d \kappa \\
& =\left[1+f_{0} \frac{\left(\frac{\omega}{C_{s}}\right)^{2}}{\sqrt{\left(\kappa^{*}\right)^{2}-\left(\frac{\omega}{C_{s}}\right)^{2}}} \cos \kappa^{*} x\right] 2 v_{i n} \cdots .
\end{aligned}
$$

where $f_{0}$ is the height of sinusoidal topography of free surface, and $\kappa^{*}=2 \pi / L$ is the horizontal wavenumber of sinusoidal topography of free 


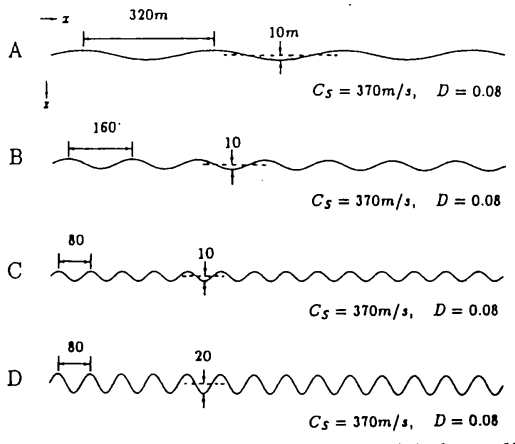

Fig.6 The half space ground models with four different sinusoidal topographies used in the examination of accuracy of the perturbation method

Table 1

\begin{tabular}{ccc}
\hline Model & Height $(m)$ & Wave Length $(m)$ \\
\hline A & 10 & 320 \\
B & 10 & 160 \\
C & 10 & 80 \\
D & 20 & 80 \\
\hline
\end{tabular}

surface with $L$ being the periodicity length (wave length) of sinusoidal topography. In the case of a plane free surface, $f_{0}=0$, and then the frequency response displacement is constantly $2 v_{\text {in }}$ in every position $x$. In the case of sinusoidal free surface the spatial variation of the frequency response displacement is sinusoidal $\left(\cos \kappa^{*} x\right)$ which is the same as the spatial variation of free surface topography since the perturbation approach in this study truncates the higher order terms. In Eq.(32), for the frequency that satisfies the condition $\kappa^{*}=$ $\omega / C_{s}$, the response displacement approaches infinity. However this phenomena is caused by neglecting the higher order scattering waves. To remove this unrealistic higher response to the frequency $\omega=\kappa^{*} C_{s}$, material damping is taken into consideration in Eq.(32), even though the half space ground model used in the computation by the boundary element method has no material damping. In the comparison the material damping ratio is assumed to be $D=0.08$ in both methods.

In the perturbation method the analytic expression is obtained by neglecting terms of order higher than irregular height $f$ and slope $\partial f / \partial x$. Therefore, the height and the slope are the key parameters for the bounds of accuracy of the perturbation method. In this comparison, the parameters $\alpha$ and $\beta$ are introduced which correspond to the relative height and the approximate slope defined as :

$$
\alpha=\frac{f_{\max }}{S_{\text {wavelength }}}=\frac{f_{\max } \omega}{2 \pi C_{s}}, \beta=\frac{4 f_{\max }}{L}
$$

where $f_{\max }$ is the maximum height of irregular surface, $C_{s}$ is the $S$-wave velocity, and $L$ is the minimum periodicity length of irregular surface. In the models used in the comparisons, $\beta=0.125$, $0.25,0.50$, and 1.00 , since $f_{\max }=10,20 \mathrm{~m}$, and $L=$ 80,160 , and $320 \mathrm{~m}$ are used (Table 1). By changing the excitation frequency in the ground model with each slope parameter $\beta$, ten cases with different height parameter $\alpha$ are computed, i.e, $\alpha=0.02$, $0.06,0.10,0.12,0.14,0.16,0.18,0.20,0.22$, and 0.24 .

In order to facilitate the comparison of the perturbation method and the boundary element method, the following two $L_{2}$ norm differences are computed :

$$
\begin{aligned}
& A B S_{L_{2} \text { Error }}=100 \sqrt{\frac{\sum_{x=1}^{n}\left(\left|v_{B}\right|-\left|v_{P}\right|\right)^{2}}{\sum_{x=1}^{n}\left|v_{B}\right|^{2}} \cdots \cdots} \\
& R E_{L_{2} \text { Error }}=100 \sqrt{\frac{\sum_{x=1}^{n}\left[\operatorname{Re}\left(v_{B}\right)-\operatorname{Re}\left(v_{P}\right)\right]^{2}}{\sum_{x=1}^{n} \operatorname{Re}\left(v_{B}\right)^{2}}}
\end{aligned}
$$

where $v_{B}$ is the frequency response displacement by the boundary element method, $v_{P}$ is the frequency response displacement by the perturbation method, and $R e$ represents the real part of the complex variable.

The two $L_{2}$ norm differences are plotted in Fig.7 against ten height parameters $\alpha$ for the four slope parameters $\beta . L_{2}$ norm differences increase rapidly when increasing the height parameter and the slope parameter. In this particular example it may be acceptable for the values of $\alpha \leq 0.10$ and $\beta \leq 0.25$. Therefore, the perturbation solution may be acceptable for the following ranges :

$$
f_{0} \leq \alpha \lambda, \quad f_{0} \leq \frac{\beta}{4} L, \alpha=0.1, \quad \beta=0.25
$$

where $f_{0}$ is the height of sinusoidal topography of free surface, $\lambda$ is the $\mathrm{S}$-wave length, and $L$ is the periodicity length (wave length) of sinusoidal free surface topography. Fig. 8 shows two examples of results of comparison for the two height parameter $\alpha=0.06,0.10$, with constant slope parameter $\beta=$ 0.25 . It is observed from Fig. 8 that the differences in both methods are small.

\section{FREQUENCY WAVENUMBER SPECTRUM OF STOCHASTIC SH WAVE}

By assuming that the incident $\mathrm{SH}$ wave motion is the stationary stochastic process and the fluctuation of irregular free surface is the stochastic field with zero mean, eventually the response displacement wave field may be a stationary and homogeneous stochastic wave. Then, its frequency wavenumber 

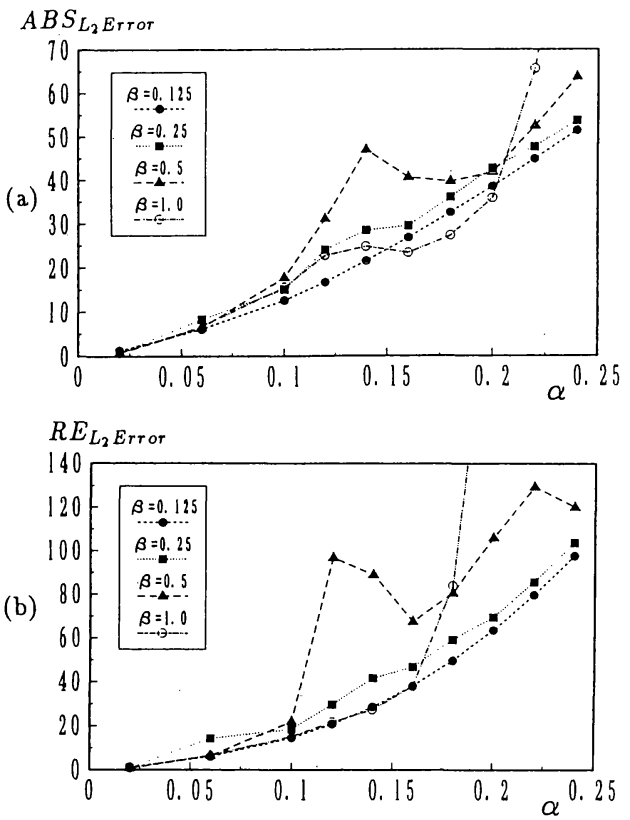

Fig.7 $L_{2}$ norm comparison of the perturbation method and the boundary element method $A B S_{L_{2} \text { Error }}$; (b) $R E_{L_{2} \text { rror }}$

spectrum is obtained as :

$$
S_{v v}(\kappa, \omega)=|v(\kappa, \omega)|^{2}
$$

Substitution of Eq.(31a) into Eq.(36) yields :

$$
\begin{aligned}
S_{v v}(\kappa, \omega) & =\left[|A|^{2} \delta\left(\kappa-\kappa_{0}\right)\right. \\
& \left.+|B|^{2} S_{f f}\left(\kappa-\kappa_{0}\right)\right] S_{\text {vinvin }}(\omega) \cdots
\end{aligned}
$$

where $S_{f f}$ is the wavenumber spectrum of the fluctuation of irregular free surface $f(x)$. The quantities $A$ and $B$ appearing in Eq.(37) are given by :

$$
\begin{aligned}
A & =\frac{2 p}{\cos \gamma H} \ldots \ldots \ldots \ldots \ldots \ldots \ldots \ldots \ldots \ldots \ldots \ldots \ldots \ldots \\
B & =\frac{2 q \gamma_{0} \sin \gamma H}{\cos \gamma H \cos \gamma_{0} H}\left(\frac{\kappa \kappa_{0}-\kappa_{0}^{2}}{\gamma \gamma_{0}}-\frac{\gamma_{0}}{\gamma}\right)
\end{aligned}
$$

Where $p$ and $q$ are given in Eqs.(31b) and (31c), and $\gamma_{0}$ corresponds to the value of $\gamma$ when $\kappa=\kappa_{0}$. The validity ranges of Eq.(37) may be estimated from Eq.(35) by changing $f_{0}$ and $L$ to the RMS (root mean square) height and the mean wave length of stochastic field $f(x)$ which are obtained from the wavenumber spectrum moments of stochastic field $f(x)$ such as :

$$
\begin{gathered}
f_{R M S}=\sqrt{\int_{-\infty}^{\infty} S_{f f}(\kappa) d \kappa} \ldots \ldots \ldots \ldots \\
L_{M E A N}=2 \pi \sqrt{\frac{\int_{-\infty}^{\infty} S_{f f}(\kappa) d \kappa}{\int_{-\infty}^{\infty} \kappa^{2} S_{f f}(\kappa) d \kappa}}
\end{gathered}
$$

where $f_{R M S}$ is the RMS height of $f(x)$, and $L_{M E A N}$ is (a)

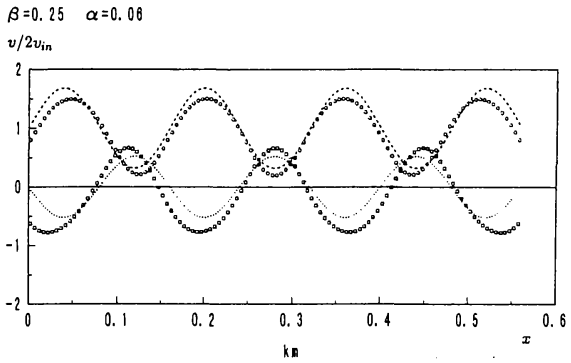

$\beta=0.25 \quad \alpha=0.1$

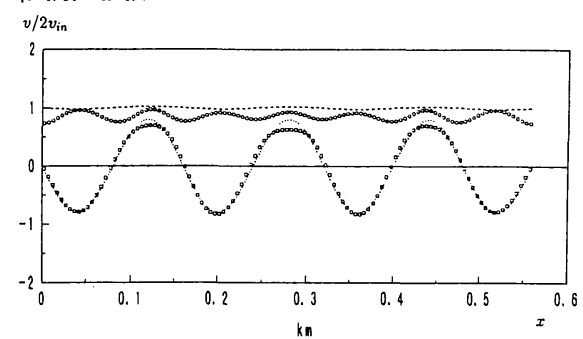

Fig.8 Examples of the frequency responses along the sinusoidal surface computed by the perturbation method (dotted lines) and the boundary element method (discrete points) : (a) $\alpha=0.06$; (b) $\alpha=0.1$

the mean wave length of $f(x)$.

\section{CONCLUSIONS}

In this paper, a closed form analytic expression of the frequency wavenumber spectrum is established. The corresponding earthquake ground motion is produced by the earthquake response of a single soil layer of irregular thickness resting on half space. The earthquake motion, assumed by a single plane $\mathrm{SH}$ wave, is transmitted to the soil layer from the half space. The analytic expressions of the earthquake responses of irregular ground are based on the perturbation method, and their accuracy is examined by comparison with the responses computed by the direct boundary element method for many rough surface models consisting of topography with sinusoidal shape. The error in the perturbation method is acceptable for the height of sinusoidal topography of less than about 10 percent of the shortest S-wave length and the slope of less than about 0.25. Finally, an analytic expression for the frequency wavenumber spectrum of the stochastic wave at irregular ground surface is then derived. Although the present paper considers the inhomogeneity of local soil layer caused by the lateral variation of the thickness of soil layer only, the effect of the inhomogeneity caused by the spatial variation of soil properties on the ground motions can be also studied using the perturbation method presented in this paper (Harada et al., 1990). The sample stochastic wave form can be efficiently simulated on the basis of the 
spectral representation method where the simulated wave consists of the superposition of a number of plane waves having amplitudes consistent with the frequency wavenumber spectrum (Shinozuka et al., 1987).

\section{ACKNOWLEDGEMENT}

The author wishes to thank Miyazaki University graduate students Satoshi Teramoto and Wilson Gorges for their kind assistance in carrying out, respectively, the numerical computations and editing necessary for the completion of the manuscript.

\section{REFERENCES}

1) Abrahamson, N. A. : Estimation of Seismic Wave Coherency and Rupture Velocity Using The SMART-1 Strong-Motion Array Recordings, UCB/EERC, 85/02, Earthquake Engineering Research Center, University of California, Berkeley, 1985.

2) Abrahamson, N. A., Schneider, J. F. and Stepp, J. C. : Empirical Spatial Coherency Functions for Application to Soil-Structure Interaction Analysis, Earthquake Spectra, Professional Journal of the Earthquake Engineering Research Institute, Vol.7, No.1, pp.1 27, 1991.

3) Bochner, S. : Lectures on Fourier Integrals, Annals Mathematical Studies, No.42, Princeton University Press, 1956.

4) Bouchon, M. : Discrete Wavenumber Representation of Elastic Wave Fields in Three-Space Dimensions, Journal of Geophysical Research, Vol.84, pp.3609 3614, 1979.

5) Harada, T. : Probabilistic Modeling of Spatial Variation of Strong Earthquake Ground Displacements, Proceedings of 8th World Conference on Earthquake Engineering, Prentice Hall, pp.605 612, 1984.

6) Harada, T. and Fugasa, T. : Characteristics of Seismic Responses of 3-Dimensional Ground with Stochastic Soil Properties, Memoirs of the Faculty of Engineering Miyazaki University, No.36, September, 1990.

7) Harada, T. and Shinozuka, M. : Ground Deformation Spectra, Proceedings of 3rd U. S. National Conference on Earthquake Engineering, Earthquake Engineering Re- search Institute, pp.2191 2202, 1986.

8) Harichandran, R. : Local Spatial Variation of Earthquake Ground Motion, Earthquake Engineering and Soil Dynamics-Recent Advances in Ground Motion Evaluation, edited by Lawrence Vom Thun, J., Geotechnical Special Publication, No.20, ASCE, pp.203 217, 1988.

9) Kashima, N., Kawashima, K., Harada, T., Isoyama; R. and Masuda, S. : Soil-Structure Interaction and Its Implication for Seismic Design of Structures, Proceedings of 9th World Conference on Earthquake Engineering, Prentice Hall, pp.605 612, 1984.

10) Kawaguchi, K., Masuda, S., Isoyama, R. and Saeki, M. : Aseismic Design of Akashi Kaikyo Bridge Foundations, Proceedings of New Zealand Japan Workshop on Base Isolation of Highway Bridges, Technology Research Center for National Land Development, pp.52 63, 1987.

11) Kennett, B. L. N. : Seismic Wave Scattering by Obstacles on Interfaces, Geophysical Journal of the Royal Astronomical Society, Vol.28, pp.249 266, 1972.

12) Loh, C. H., Penzien, J. and Tsai, Y. B. : Engineering Analysis of SMART-1 Accelerograms, Earthquake Engineering and Structural Dynamics, Vol.10, pp.579 591, 1982.

13) Public Works Research Institute : A Proposal for Earthquake Resistant Design Method, Technical Memorandum of PWRI, Ministry of Construction, No.1185, March 1977 (in Japanese).

14) Shinozuka, M., Deodatis, G. and Harada, T. : Digital Simulation of Seismic Ground Motion, Stochastic Approaches in Earthquake Engineering, edited by Lin, Y. K. et al., Springer-Verlag, pp.252 298, 1987.

15) Trifunac, M. D. : Scattering of Plane SH Waves by a Semicylindrical Canyon, Earthquake Engineering and Structural Dynamics, Vol.1, pp.267 281, 1973.

16) Wong, H. L. and Jennings, P. C. : Effect of Canyon Topography on Strong Ground Motion, Bulletins of Seismological Society of America, Vol.65, pp.1239 1257, 1975.

17) Yuan, X. and Men, F. L. : Scattering of Plane SH Waves by a Semicylindrical Hill, Earthquake Engineering and Structural Dynamics, Vol.21, pp.1091 1098, 1992.

(Received August 6, 1993)

\section{地震動の SH 確率波モデル}

\section{原田隆典}

半無限地盤と不整形な地表面を有する表層地盤から成る 2 層地盤に $\mathrm{SH}$ 波が入射する 場合の応答を摄動法により解析的に求め，地震動の振動数-波数スペクトルを推定した． 解析解の精度と適用限界条件式を直接境界荌素法から導いた。 